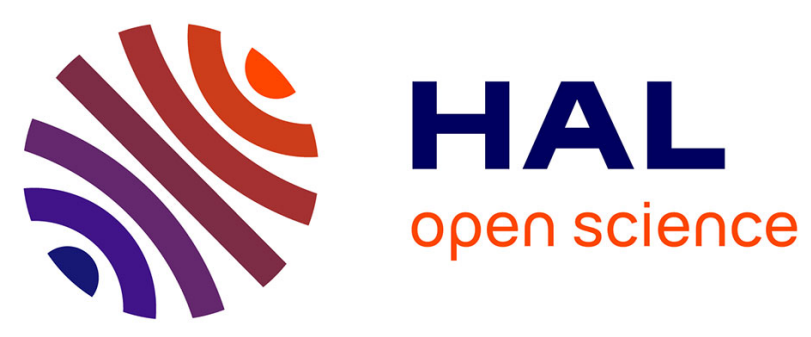

\title{
Pouvoirs des infrastructures
}

Nathalie Roseau

\section{To cite this version:}

Nathalie Roseau. Pouvoirs des infrastructures. Histoire urbaine, 2016, 45, pp.5-16. 10.3917/rhu.045.0005 . hal-01339805

\section{HAL Id: hal-01339805 https://hal.science/hal-01339805}

Submitted on 30 Jun 2016

HAL is a multi-disciplinary open access archive for the deposit and dissemination of scientific research documents, whether they are published or not. The documents may come from teaching and research institutions in France or abroad, or from public or private research centers.
L'archive ouverte pluridisciplinaire HAL, est destinée au dépôt et à la diffusion de documents scientifiques de niveau recherche, publiés ou non, émanant des établissements d'enseignement et de recherche français ou étrangers, des laboratoires publics ou privés. 


\section{INFRASTUCTURES URBAINES}

NATHALIE RoseAU*

\section{Pouvoirs des infrastructures}

$\mathrm{D}$ ans Les villes dans la France Moderne (1740-1840), Bernard Lepetit évoquait l'édification du réseau routier en France au cours du $\mathrm{XVIII}^{\mathrm{e}}$ siècle dont il démontrait combien elle avait été influencée par le système urbain préexistant tout en assurant le décloisonnement du territoire. Etudiant le processus de mise en ouvre de la nouvelle carte administrative du pays sous la Révolution, il montrait aussi combien, en dépit de sa rupture avec l'Ancien Régime, cette nouvelle armature s'était nourrie aux modes anciens de fonctionnement économique des réseaux de chefslieux. Quelques années plus tard, dans un article posthume revenant sur deux décennies d'histoire urbaine, l'historien concluait que «ces deux formes d'aménagement du territoire (la route et l'administration territoriale) permettent de souligner la manière complexe dont le système urbain participe à son propre devenir, dont il est à la fois, pour utiliser le vocabulaire de la statistique, une variable explicative et expliquée [...]. Ainsi, en produisant du neuf avec le vieux et du vieux avec le neuf, le système urbain est en permanence dans un déséquilibre partiel. Il est à tout instant engagé dans un processus historique de transformation de luimême ${ }^{1}$.

C'est à l'étude de ces processus qu'invite ce numéro d'Histoire Urbaine dont les contributions analysent, à des moments différents de leur histoire, plusieurs des équipements qui aménagent et transforment les villes. Des installations portuaires ou fluviales à la carte des lycées en passant par les fortifications ou les réseaux de tramways: c'est sous le terme d'infrastructure que les éditeurs ont souhaité rassembler ces ouvrages, car ils parta-

* École des Ponts ParisTech, Laboratoire Techniques, territoires et sociétés (LATTS).

1. Bernard Lepetit, «La ville: cadre, objet, sujet. Vingt ans de recherches françaises en histoire urbaine. ", Enquête, 4, La ville des sciences sociales, 1996, https://enquete.revues.org/663, p. 10-11 ; idem, Les villes dans la France moderne (1740-1840), Paris, Albin Michel, 1988.

H.U. $\mathrm{n}^{\mathrm{o}} 45$ - avril 2016 - p. 5 à 16 
gent un même dessein : former un soubassement par lequel la ville et le territoire se modernisent. Si certains sont plus visibles que d'autres, ils se qualifient tous par leur grande dimension, une échelle qui dépasse en même temps qu'elle épouse celle de leur ville, les plaçant au centre d'un écheveau de pouvoirs.

Ce terme commun permet aussi de s'inscrire dans un champ de recherche, qui a déjà été travaillé de diverses façons, l'infrastructure constituant depuis quelques décennies un objet d'histoire à part entière ${ }^{2}$. Car, non seulement, on ne peut comprendre l'histoire des villes sans comprendre la construction historique des infrastructures qui les façonnent, mais l'histoire des infrastructures - parce qu'elle soulève des questions propres relatives aux sources, aux terrains, aux objets et aux méthodes de l'histoire - enrichit la manière de faire de l'histoire urbaine. Ainsi, si l'on pense à la façon dont se transforment au XIX ${ }^{\mathrm{e}}$ siècle les nécropoles parisiennes - sous l'effet de leur municipalisation, de leur aménagement comme cimetière-jardin, de leur mobilisation dans le cadre d'événements commémoratifs, des règlementations funéraires -, on entrevoit peu à peu se dessiner un dispositif au sens que lui donnait Michel Foucault d'un réseau qu'il est possible de tracer entre les différents éléments $d$ '«un ensemble résolument hétérogène, comportant des discours, des institutions, des aménagements architecturaux, des décisions règlementaires, des lois, des mesures administratives, des énoncés scientifiques, des propositions philosophiques, morales, philanthropiques, bref du dit aussi bien que du non dit $»^{3}$. Envisagées comme dispositifs, l'étude des infrastructures peut éclairer de manière fructueuse, non seulement les conditions selon lesquelles les villes se sont modernisées, mais

\footnotetext{
2. Nous n'avons pas ici la place pour établir un état de l'art, national et international, des travaux relatifs à ce courant d'études. Toutefois, nous souhaiterions citer un panel d'ouvrages qui montre les dimensions multiples - politique, économique, sociale, écologique, territoriale et architecturale - que revêt l'étude des infrastructures dans le champ de l'histoire urbaine. JeanLouis Cohen et André Lortie, Des fortifs au périph, Paris, Les seuils de la ville, Paris, Picard/ Editions du Pavillon de l'Arsenal, 1992; Marc Desportes et Antoine Picon, De l'espace au territoire, L'aménagement en France (XVI ${ }^{e}-X X^{e}$ siècles), Paris, Presses de l'École Nationale des Ponts et Chaussées, 1997; Sabine Barles, La ville délétère, Médecins et ingénieurs dans l'espace urbain, $X_{\text {XIII }}{ }^{-}$-XIX ${ }^{e}$ siècles, Champ Vallon, 1999; Mathew Gandy, Concrete and clay, reworking nature in New York City, MIT Press, 2002; Karen Bowie et Simon Texier (sous la direction de), Paris et ses chemins de fer, Délégation à l'Action historique de la ville de Paris, 2003; Eric Alonzo, Du rondpoint au giratoire, Marseille, Editions Parenthèses, 2005 ; Denis Bocquet, Samuel Fettah (sous la direction de), Réseaux techniques et conflits de pouvoir, Les dynamiques historiques des villes contemporaines, Collection École Française de Rome, 2007; Stéphanie Sauget, À la recherche des pas perdus, Une histoire des gares parisiennes, Paris, Tallandier, 2009; Nathalie Roseau, Aerocity, Quand l'avion fait la ville, Marseille, Editions Parenthèses, 2012.

3. Michel Foucault, «Le jeu de Michel Foucault», Entretien Dits et écrits, Tome II, Paris, Gallimard, 1994 [1977], p. 299.
} 
aussi les médiations selon lesquelles les villes ont pensé et réalisé leur infrastructure.

«Variable explicative et expliquée»: c'est dans cette perspective qu'il convient d'apprécier les contributions qui suivent comme autant de jalons s'inscrivant dans l'appel de Bernard Lepetit à considérer l'histoire urbaine comme «histoire problème». En cela, ce numéro permet de poursuivre l'observation de l'évolution des infrastructures dans les milieux urbains et du jeu d'influences entrecroisées qu'elles nouent avec les villes dont elles sont les instruments de leur transformation. Il contribue à enrichir la question du déterminisme en histoire, invitant à renoncer à l'idée d'une causalité purement sociale ou technique au profit d'une causalité plus complexe et multiple. Car, comme le rappelait Marc Bloch, «il n'existe point de train d'ondes causales privilégié; point d'ordre de faits toujours et partout déterminants, opposés à de perpétuels épiphénomènes; au contraire, toute société, comme tout esprit, est issue de constantes interactions ${ }^{4}$.

\section{Circonscrire l'infrastructure}

Les éditeurs ont pris le pari heuristique de faire coexister dans un même ouvrage des questions et des points de vue aussi variés que la construction des villes neuves fortifiées dans la Flandre de Charles Quint, la naissance des villes de la Meuse moyenne jusqu'au XVII ${ }^{\mathrm{e}}$ siècle, l'aménagement portuaire de la côte basse-languedocienne aux XVII ${ }^{\mathrm{e}}$ et XVIII ${ }^{\mathrm{e}}$ siècles, la consolidation du réseau urbain national des lycées d'Etat de 1830 à 1880 , le rôle des comités de quartier dans l'extension au tournant du siècle dernier du réseau de tramway marseillais. Ce panel éclectique dévoile diverses faces de l'infrastructure.

Le dossier s'ouvre sur l'étude de l'urbanisme des villes d'Hesdin, Philippeville et Charlemont, ville neuves créées au milieu du Xvi ${ }^{\mathrm{e}}$ siècle à la frontière entre la France et les Pays-Bas espagnols, que Nathalie Dereymaeker analyse comme projets à la fois guerriers et esthétiques, dont les contraintes géographiques et pratiques orientent l'édification concrète. En retraçant la naissance des villes mosanes de Basse Lotharingie, de Maastricht à Mézières, des origines de leur fondation au premier siècle jusqu'au $\mathrm{XVII}^{\mathrm{e}}$ siècle, Marc Suttor raconte d'une part leur construction progressive en rapport avec le fleuve et montre d'autre part comment s'articule le développement conjoint des villes mosanes dont les pouvoirs relatifs

4. Marc Bloch, "Technique et évolution sociale: réflexions d'un historien», Europe, 1938, Mélanges historiques, Paris, Sevpen, 1963, tome II, pp. 837-838. 
consolident la Meuse comme territoire en soi. Avec l'étude des villes maritimes intérieures de la côte basse du Languedoc de l'époque moderne, Stéphane Durand montre que, dès le XVII ${ }^{\mathrm{e}}$ siècle, et du fait d'une configuration territoriale particulière, les aménagements portuaires civils dépassent l'échelle des villes, mobilisant des rapports conflictuels de pouvoirs entre le royaume, les villes et l'assemblée des états du Languedoc. Explorant le processus qui, de 1830 à 1880 , conduit à la transformation de 45 collèges communaux en lycées d'Etat, ainsi que les rapports de forces politiques, économiques, symboliques à l'œuvre, Solenn Huitric s'intéresse au lycée comme attribut de la puissance administrative et politique des villes - comme pourrait l'être aujourd'hui l'université. Le rôle des Comités d'Intérêt de Quartier dans les projets d'extension du réseau de tramway marseillais, mis à jour par Hisao Kokubu, illustre enfin le processus selon lequel ces formes associatives se sont constituées et fédérées en référence aux problèmes d'urbanisation, tout en jouant un rôle déterminant dans la formation du réseau de transport.

Par les cinq regards obliques qu'il suggère, ce panel montre que l'infrastructure se laisse difficilement saisir comme un objet aux contours clairs. Et même lorsque ceux-ci se précisent, leur nature fluctuante les dissout dans d'autres formes. Les études de cas montrent ainsi le caractère doublement volatil et structuré des objets décrits, dont l'échelle est ample, par leur taille, l'étendue de leur déploiement, leur capacité d'expansion ou d'émancipation.

La difficulté à saisir les contours de l'objet procède aussi de sa capacité propre d'assimilation dans l'espace de la ville. Lorsqu'elle parvient à son but - irriguer, équiper, aménager -, l'infrastructure semble se fondre dans la ville, cette invisibilité relative témoignant tout autant du succès de sa mise en œuvre que des processus d'hybridation à l'œuvre. Ainsi, revenant au cas exposé par Nathalie Dereymaeker, on voit combien la conception des villes neuves fortifiées procède d'une coopération étroite entre art militaire et art urbain, un art stratégique de la conquête et de la défense d'une part, et un art anthropomorphique de la géométrie et de la perspective d'autre part. De cette acculturation réciproque, s'inventent non seulement un système spatial - composé d'un mur continu, de remparts, courtines, bastions, places d'armes - mais aussi toute une structure qui fonde la ville - forme, parcellaire et plan.

Si l'on veut, comme nous y invitait Bernard Lepetit, «échapper aux deux risques symétriques d'une complexification opacifiante et d'un évanouissement de l'objet ${ }^{5}$, il nous faut donc régler nos focales, armer nos 
concepts, préciser le vocabulaire. Dans cette perspective, nous pourrions envisager l'infrastructure selon une triple acception: matérielle, l'infrastructure se composant de divers ouvrages physiques, banals ou exceptionnels, de plusieurs ordres et échelles, formant une strate territoriale qui épouse et transcende l'espace urbain; interactionnelle, l'infrastructure constituant un objet de médiation faisant agir et interagir des moteurs et des pouvoirs, inscrits dans un contexte situé, qui la façonnent, la déstabilisent et la re-stabilisent; imaginaire, l'infrastructure étant le produit d'une pensée - nourrie par des valeurs, des désirs et des attentes, traduite dans les projets, les doctrines et les savoirs - et la source de représentations urbaines. Les articles qui suivent nous semblent riches à observer selon ce triptyque, la question des pouvoirs pouvant constituer un fil tendu pour relier ces jalons. Ils sont incontournables à la fois pour motiver la naissance d'une infrastructure, la pérenniser et la faire agir comme instrument de la puissance des villes. Deux prismes nous guideront dans l'exploration de cette voie: la question des échelles et celle des causalités.

\section{La transcendance des échelles}

Les articles décrivent d'abord ce mouvement ininterrompu selon lequel l'infrastructure s'affranchit en même temps qu'elle façonne l'espace de la ville, le déformant, le reconfigurant. Cette dynamique ne se fait pas sans heurts, cristallisant des ambitions et des conflits. Surtout, cette situation à la frontière positionne l'infrastructure dans une sorte de schizophrénie structurelle dont les études qui suivent nous permettent de cerner trois caractéristiques: l'appartenance à un intermonde, un savoir spécifique, l'iconicité.

Observant le système urbain de la Meuse moyenne, Marc Suttor montre que les villes mosanes évoluent dans un double rapport d'échelle par rapport au fleuve. Les villes se créent chacune dans une relation nourricière au fleuve; leurs pouvoirs commerciaux, politiques, épiscopaux, vont croissant, donnant naissance à une ville système, la Meuse urbaine. En Bas-Languedoc, c'est dès l'origine de la naissance portuaire du territoire que l'infrastructure se construit de manière extraterritoriale aux villes. Stéphane Durand décrit cette situation duale du territoire de l'infrastructure, à cheval sur plusieurs échelles auxquelles elle n'appartient jamais pleinement. Du fait de la configuration particulière des lieux, qui impose d'aller «chercher » la mer depuis les ports intérieurs, et de « remonter " les terres pour gagner les villes maritimes, les aménagements infrastructurels se déploient dans un intermonde entre la ville et la mer, constitué d'avant- 
ports et d'ouvrages - môle, canaux, digues, quais, graus. À partir de l'extrême fin $\mathrm{du} \mathrm{XVI}{ }^{\mathrm{e}}$ siècle, l'échelle de l'infrastructure suppose des politiques d'aménagement de grande ampleur qui doivent répondre à la nécessité de penser l'interface entre un espace de navigation intérieure et les infrastructures aménagées sur la côte, et dont la portée transcende les échelles proprement urbaines. En retour, l'extraterritorialité des ports agit sur la richesse urbaine car elle permet aux villes d'accroître leur rayonnement et leur pouvoir.

S’il est situé au cœur des villes, le lycée, envisagé comme instrument de rayonnement départemental, incarne aussi cette exception qui ancre et distingue la ville dans un réseau supra-urbain. Les mobilisations des édiles qui entendent défendre ou rehausser le statut de leur ville, montrent toute l'importance que revêtent ces équipements au-delà de leur fonction proprement urbaine. Solenn Huitric souligne de quelle façon leur dimension supra-territoriale les inscrit dans un réseau urbain qui les fait exister, mais surtout nationalise leurs enjeux locaux. Dans un autre registre, les conflits qui se nouent autour de l'extension du réseau de tramway marseillais, mettent à jour le jeu d'échelles auquel celui-ci appartient. Avec le corpus de délibérations, d'enquêtes, de campagnes de presse, Hisao Kokubu nous fait comprendre comment se positionnent les pouvoirs autour d'enjeux qui les mobilisent, tels que l'équité sociale sur le territoire de l'agglomération («le tram à dix sous» partout), les rapports entre centre et périphérie. En retour, on voit de quelle façon la réalisation $\mathrm{du}$ réseau contribue à institutionnaliser des structures qui à l'origine n'étaient qu'embryonnaires. Face aux enjeux urbains, les comités d'intérêt de quartier, de simples chambres locales d'enregistrement de plaintes individuelles, se fédèrent progressivement et vont peser comme contrepouvoirs d'agglomération.

Conséquence de cette extraterritorialité, la naissance et le développement d'une infrastructure nécessitent des savoirs spécifiques que les villes ne possèdent pas toujours, en tout cas pas complètement du fait des échelles inédites, spatiales, techniques, financières, auxquelles elles sont confrontées. Des questions nouvelles se posent, qui exigent d'être raisonnées au-delà des villes. Des idées circulent, des modèles sont mobilisés, des compétences se forment, des figures professionnelles émergent, des élites urbaines se professionnalisent. Le cas de l'édification des fortifications, raconté par Nathalie Dereymaeker, illustre le processus précoce d'internationalisation croisée dont font l'objet la conception et les modalités de réalisation des ouvrages défensifs urbains. Le portrait de Sebastian van Noyer, auteur du projet des trois villes neuves, à la fois ingénieur, artiste et architecte, relate ses voyages à Rome et en Italie, dont il ramène des écrits, 
des cartes et des vues; il trace aussi le réseau de relations auquel appartient le maitre d'œuvre, entre commanditaires et formations. C'est dans cet entrelacs de connaissances et d'influences issues de l'art militaire, de l'architecture et de l'art urbain, de l'ingénierie et de la science du chantier, qu'il se forme et contribue à l'édification des villes flamandes. Villes dont les ingénieurs italiens, partis pour conseiller leurs collègues flamands, découvriront les ressorts, adaptés à la situation géographique du Nord de l'Europe, et dont ils apprendront à leur tour.

C'est aussi aux ingénieurs des fortifications que sera confié l'urbanisme de l'avant-port de Sète dont les édifices et les ouvrages, du canal de communication à la ville portuaire, supposent des compétences qui dépassent celles des villes. Mais, si les villes sont façonnées par des modèles, des doctrines, des savoirs, des techniques, des corps de métiers qui ne leur sont pas propres, elles comprennent vite l'intérêt qu'il y a à se les approprier, la réalisation de l'infrastructure, défi hors norme, leur permettant de passer un cap. Elles doivent parfois prouver, comme dans le cas des lycées, leur capacité à s'élever pour obtenir les faveurs nationales, les subsides de l'Etat, et exister dans la concurrence que se livrent les cités; elles y voient souvent leur intérêt, maîtrisant leurs ouvrages, accroissant leur crédit vis à vis de leurs administrés, affirmant leur autorité, comme à Marseille, dans les relations qu'elles nouent avec les autres acteurs techniques ou gestionnaires du tramway urbain.

Que produisent ces entrecroisements d'échelles qui appartiennent à des registres territoriaux et disciplinaires différents? Au-delà (et sans doute en raison) de la complexité des échelles et des savoirs que sa conception et son évolution mobilisent, la figure de l'infrastructure anticipe et compense la perte de netteté géographique et de clarté politique dont le territoire va peu à peu pâtir. Figure abstraite dans son dessin, figure concrète dans sa matérialisation, l'infrastructure devient peu à peu un étendard de ralliement, icône urbaine qui condense et renforce en même temps la représentation de la ville qu'elle structure. Nathalie Dereymaeker décrit ainsi l'enceinte comme le produit d'une vision militaire dont le penchant pour la rigueur d'exécution trouve dans la ville idéale de la Renaissance, le modèle qui s'accorde. De l'association de l'art de la guerre à l'art de la ville, naît une figure stratège dont la géométrie, les perspectives et les proportions inscrivent les ouvrages dans la cité qui l'incarne. Soumettant à sa logique, le plan et la forme, les espaces libres et le parcellaire, elle modèle la cité en définissant son langage. En même temps, si la morphologie de la ville découle du dispositif de l'infrastructure, celui-ci s'accorde selon des références et des échelles urbaines. Urbanisation de l'infrastructure ou infrastructuralisation de la ville? Les vues assimilent la forme de la 
cité à celle de l'enceinte, l'infrastructure devenant l'image de la ville. Jules Romains ne dira-t-il pas au début du siècle dernier, à propos de Paris et de sa dernière enceinte, qu' " après lui avoir servi de protection avancée, elle était devenue la forme même de le la ville " ${ }^{6}$. Nouvelle limite de la ville, la fortification en dessine les contours et anticipe son extension.

En d'autres cas, le processus peut être moins radical mais tout aussi structurant, comme l'exprime le cas de la Meuse. Marc Stuttor montre bien la transformation du fleuve qui, de géographie naturelle, s'équipe et se constitue comme infrastructure aux ramifications étendues, pour finir par incarner non seulement l'image de la ville mais surtout l'image d'un réseau urbain qui la prend pour colonne vertébrale. La sémantique est ici intéressante pour comprendre ce processus d'incarnation, qu'il s'agisse des noms de villes (Maastricht, Mosae Trajectus), des ouvrages - ordinaires ou non - composant la structure sur laquelle se bâtiront les villes - "applé», gué, jetée, pont, débarcadère, port, moulin -, des caractères urbains qui naissent des équipements liés au fleuve ou des métiers les «mairins » et les «mairniers ». L'âme des villes inscrite dans leur toponymie, se lit selon ce soubassement de la relation du sol au fleuve.

\section{La matrice des causalités}

En décryptant de manière fine les processus temporels par lesquels les infrastructures se projettent et s'assimilent, ces études montrent l'enchevêtrement d'un faisceau de moteurs géographiques, techniques, politiques, économiques, sociaux, qui éloigne des déterminismes trop simplistes, et entrecroise différentes causalités dont les processus historiques évoluent les uns par rapport aux autres. Ces fils noués et dénoués forment une toile dont nous allons maintenant cerner quelques nœuds structurants.

D'abord, ce faisceau de causalités gagne à être mis en récit par le double mouvement selon lequel se crée l'infrastructure dans l'espace et le temps, faisant l'objet d'un cycle ininterrompu de création, adaptation, émancipation, obsolescence, reconversion. Qu'il s'agisse des villes mosanes ou de la côte languedocienne, les infrastructures stimulent le développement urbain (implantation, développement, assise, modernisation) en même temps qu'elles s'en nourrissent (commerce, pouvoirs politique et religieux, industrie, défense). Leur position à cheval, dans les interstices des mondes

6. Jules Romains, Les Hommes de bonne volonté, Livre I, «Le 6 octobre», Robert Laffont, 1988 [1932], p. 111 . 
techniques, politiques et urbains, les conduit à évoluer dans un processus que Stéphane Durand qualifie comme dynamique d'ajustement. Les aménagements portuaires languedociens se construisent dans un équilibre en mouvement qui entrecroise le pouvoir royal, les villes et l'assemblée des états du Languedoc, dont les intérêts et les leviers sont de divers ordres et dont les actions, conjuguées ou non, orientent la trajectoire des lieux. Ainsi, c'est lorsque le développement du commerce et des techniques exige une modernisation de l'infrastructure portuaire, que les hiérarchies naturelles sont bousculées par d'autres causalités au rang desquelles figure l'influence des pouvoirs supra-urbains. Cette transformation s'intensifie sous le joug de l'intervention d'une puissance politique supérieure, opérant à grande échelle, celle du royaume, porté par le mercantilisme d'Etat de Colbert, qui raisonne à l'échelle du bassin méditerranéen et valorise le port de Sète comme élément avancé de son dispositif articulé à Marseille. L'assemblée des États du Languedoc constitue quant à elle un niveau institutionnel intermédiaire puissant, car inscrit localement en même temps qu'il dispose d'assiettes diocésaines conséquentes lui permettant de développer des politiques commerciales ambitieuses. Toutefois, si les états sont des assemblées représentatives des villes et de leurs intérêts, le choix du lieu et de la nature des investissements relève de multiples facteurs qui peuvent échapper aux villes. Ces dernières dépendent principalement de l'action du roi et de l'assemblée des états pour le développement de leurs infrastructures, qui conditionnent leur accès à la mer et le dynamisme de leurs trafics.

De même Solenn Huitric identifie quelques uns des moteurs qui vont déterminer l'élévation ou le déclassement des villes dans la course aux lycées d'État. Certains sont endogènes - tradition scolaire, position centrale, qualité des installations -, d'autres exogènes - un seul lycée par département, existence d'appuis nationaux, concurrence ou non des autres villes. Le lycée est stratégique de par son impact sur l'économie, l'attrait, le prestige. Fort de ce pouvoir que lui confère la décision d'une labellisation d'État, le ministère compare les villes, leurs chances de succès, en distingue certaines naturellement, en relègue d'autres, ou met en concurrence, suscitant un lobbying accru. Les villes ne restent pas passives, elles développent un "répertoire argumentatif commun" sur les qualités de leur candidature. La stratégie vis à vis des autorités départementale et nationale, les conduit à réordonner les priorités. Le paradoxe veut que, en intégrant le maillage tant convoité des lycées d'État, elles gagnent en importance tout en étant tributaires de ce même réseau. 
L'émergence de formes diverses de coalition des pouvoirs, visant inconsciemment ou pas, à se renforcer mutuellement pour consolider l'infrastructure, constitue un caractère récurrent des trajectoires relatées par nos auteurs. Avec l'exemple du tramway de Marseille, Hisao Kokubu montre bien la montée en puissance conjointe du projet et des comités d'intérêt de quartier de l'agglomération, dont c'est la principale revendication. Si les opposants sont peu nombreux - les cochers ou des commerçants heurtés par les nuisances ou la réduction de la voie publique -, beaucoup entendent peser dans la conduite du projet parmi lesquels les acteurs décisionnaires, municipalités, autorités concédantes, ingénieurs, mais aussi des acteurs qui se constituent pour faire valoir leurs vues - corporations, associations alors qu'ils n'ont pas de pouvoirs financiers, politiques ou techniques directs. Soulevées par ces tiers-pouvoirs, des questions vont modifier le paysage des enjeux. La lutte pour un tarif unique sur le réseau ou les conflits autour du choix des têtes de ligne, ouvrent le débat à des enjeux sociaux autour de l'équité territoriale ou du statut urbain de la périphérie. La fédération des comités d'intérêt de quartier de banlieues se bat pour un tarif unique, mobilisant ministre et conseil d'Etat par l'intermédiaire du maire de Marseille qui s'arrogera avec eux les lauriers de la victoire, en présentant la nouvelle convention comme "notre cadeau à la population". Les convergences d'intérêt peuvent aussi céder la place à des luttes d'intérêt entre quartiers, qui reflètent les statuts inégaux des différentes parties de l'agglomération. Le déplacement de têtes de lignes pénalise ceux qui les accueillaient, tandis que, pour pallier la saturation du réseau, des comités de quartier entendent réduire les prérogatives de certaines catégories de passagers. La forme associative se construit ainsi progressivement en référence aux problèmes d'urbanisation soulevés par l'extension du tramway développement du réseau, tracé des lignes, tarifications, choix des terminus intermédiaires. Cette forme fluctuante va s'affirmer dans la sphère urbaine, consolidant son pouvoir au fur et à mesure que s'opère l'urbanisation du tramway. Se forge une doctrine urbaine qui procède des enjeux soulevés par la réalisation concrète du réseau de transport et la détermine en retour.

Un acteur prédomine dont le rôle dans la genèse des infrastructures semble essentiel. Il s'agit de l'État. Certes les sites considérés se localisent en France au sein de territoires qui vont de la ville à l'agglomération, du système urbain au territoire maritime, mais précisément parce que leurs infrastructures nouent des liens de nature et d'échelle diverses, le rôle de l'État apparaît à chaque fois stratégique, emportant la décision ou la neutralisant. Et la comparaison avec la Meuse urbaine montre bien en creux, combien la situation jacobine de la France joue en faveur d'un rôle structurant de l'État sur la longue durée. Au-delà de nos frontières, le système 
urbain s'équilibre autrement, par le jeu de villes plus autonomes, plus émancipées, où l'État paraît plus effacé.

Le cas des lycées, où l'on voit que la ville se met en quatre pour défendre sa capacité à intégrer le club des élus, montre que l'attribution du label État lui permettrait en retour de rehausser son niveau. Au-delà de ce rôle de distributeur, l'État stimule la rivalité entre les villes, tout en affichant l'égalité territoriale - un par département. En l'espèce, la politique d'État remodèle ou confirme la hiérarchie urbaine, distingue et intègre les villes tout en les rendant servantes du réseau national. L'État peut être juge et arbitre, comme il peut être investisseur, à l'instar de son rôle dans la situation languedocienne, où sa puissance de feu va bouleverser les équilibres locaux et modifier le cours des choses pour les villes. Cette influence de l'État sur l'aménagement du territoire s'inscrit dans la longue durée, comme l'évoque Stéphane Durand en citant en conclusion de son article la mission Racine, structure créée en 1963 à des fins d'aménagement touristique pour conduire de grands travaux d'infrastructure en vue de développer le littoral du Languedoc-Roussillon, et qui sera en particulier à l'origine de la fondation de la station balnéaire du cap d'Agde.

\section{L'imaginaire de l'infrastructure}

$\mathrm{Au}$ fil de ce dossier, l'infrastructure est saisie dans ses dimensions multiples de projet abstrait, réalisation concrète, enjeu de pouvoirs. Revenant aux trois acceptions que nous avions avancées pour circonscrire la notion d'infrastructure, nous voyons que les deux premières acceptions, matérielle et interactionnelle, traversent les études, dans la spatialité des objets décrits et l'agencement des pouvoirs qui agissent sur eux. Par contraste, il nous semble que la troisième acception, imaginaire, gagnerait à être plus approfondie. Comprendre le rôle des représentations dans les réflexions de ceux qui conçoivent, décident, réalisent les infrastructures, permettrait de mettre à jour des périodisations parallèles et croisées, non plus celles apparentes des causalités techniques, financières, politiques, mais celles, plus latentes, de l'imaginaire qui dort, se réactive ou cristallise selon les occasions; "Le temps de l'histoire [...] est le plasma même où baignent les phénomènes et comme le lieu de leur intelligibilité », nous disait Marc Bloch7. La question des savoirs en particulier, nécessite de

7. Marc Bloch, Apologie pour l'histoire ou métier d'historien, Armand Colin, 1974 [1941], p. 36. Écouter la leçon inaugurale de Patrick Boucheron au Collège de France, "Ce que peut l'histoire", 17 décembre 2015, http://www.college-de-france.fr/site/patrick-boucheron/inaugural-lecture-201512-17-18hoo.htm (consulté le 8 février 2016). 
16 / Histoire urbaine - 45 / avril 2016

comprendre comment l'infrastructure se constitue comme objet aux règles et aux connaissances propres, instituées et pérennisées.

De la confrontation entre le passé et le présent, peuvent émerger d'autres questions pour la perspective historiographique. D'abord, le sujet infrastructurel traverse maintes disciplines des sciences sociales et humaines. Dialoguer entre disciplines sur un objet, permettrait de nourrir cette histoire à construire mais aussi de discuter certaines théories en cours. Comme nous y invite Antoine Picon, la position défendant l'avènement d'un urbanisme de la fracture à l'ère numérique, en contraste avec l'ère industrielle, doit être par exemple confrontée à l'histoire longue qui montre que les réseaux ont toujours eu des effets territoriaux à la fois intégrateurs et désintégrateurs ${ }^{8}$.

Ensuite, l'objet reste toujours d'une grande actualité pour l'urbanisme contemporain, comme l'atteste par exemple le glissement opéré des visions du Grand Paris, défendues lors de la consultation internationale d'urbanisme de 2008, au projet de supermétro du Grand Paris Express initié dans la foulée de ces esquisses. On voit ici combien l'infrastructure demeure encore l'un des remparts de l'urbanisme volontaire, accordant à la technique une fonction supérieure, qui entendrait répondre aux apories urbaines que les instruments courants ne semblent pas pouvoir vaincre. L'actualité du débat sur le financement des infrastructures gagnerait d'ailleurs à être éclairée par l'étude historique des moyens financiers dont ont fait l'objet dans le passé, les créations d'équipements - des ports aux canaux, des enceintes aux digues, des réseaux de tramways aux lycées dont les échelles dépassaient pour certains les capacités financières des villes ou des comtés. Promesse et problème en même temps: paradoxalement, alors que les questions posées montrent que le sujet s'inscrit dans une histoire de longue durée, l'objet est souvent considéré comme a-spatial et a-historique, victime d'une double amnésie. En cela, son histoire est essentielle à l'urbanisme contemporain.

8. Antoine Picon, La ville des réseaux, Un imaginaire politique, Manucius 2014. Il nuançait la position soutenue par les géographes Stephen Graham et Simon Marvin dans leur ouvrage Splintering urbanism. Networked infrastructures, technological mobilities and the urban condition, Londres, New York, Routledge, 2001. 